Published version: Baggs, E. (2021). All affordances are social: foundations of a Gibsonian social ontology. Ecological Psychology, https://doi.org/10.1080/10407413.2021.1965477

\title{
ALL AFFORDANCES ARE SOCIAL: FOUNDATIONS OF A GIBSONIAN SOCIAL ONTOLOGY
}

\author{
Edward Baggs \\ Rotman Institute of Philosophy \\ University of Western Ontario \\ ed.baggs@gmail.com
}

\begin{abstract}
Ecological psychology is built on a perception-oriented ontology. The primary focus has been on explaining the perception and action behavior of individual animals. To accommodate social phenomena within the ecological approach, it is necessary to expand the ontology, however theorists have been unclear about how to do this. The paper presents a negative argument and a positive programmatic outline. The negative argument is against the use of the term 'social affordance', a term that confuses the perspective of the researcher with that of the animal. Instead, it is advocated that we adopt, as a working hypothesis, the claim that all affordances are social; that is, all affordances are public and are, in principle, observable by a third party. The programmatic outline then shows that affordances alone are insufficient for describing social meaning. An ecological social ontology requires new tools for describing interaction processes, symbolic meaning, and material culture as structures occurring within the populated environment.
\end{abstract}

\section{Introduction}

When James J. Gibson developed his ecological approach, his motivation was to overcome the perplexities of the existing psychology of perception (Gibson, 1966, 1967b a, 1979). Gibson proposed to reject the dominant perceptual theory and to replace it with a new one. He advocated that we should reject the classical understanding of perceiving as the process of reconstructing an image or model of the external world, and replace it with a view of perceiving as a process of directly attending to meaningful structure that already exists in the world (Baggs and Chemero, 2020b). Gibson's ecological theory was a radical rethinking of the psychology of perception. According to Reed (1988), however, Gibson's ultimate ambitions were even greater than this. Reed claims that Gibson focused on the problem of perception because he believed 'that a breakthrough in the understanding of perceptual awareness and knowledge would carry in its wake a new approach to the whole of psychology' (Reed, 1988, 2). The history of ecological psychology since Gibson can be viewed in large part as an attempt to make good on this ultimate ambition (Chemero, 2009; Heft, 2013; Reed, 1996).

If the ecological approach is to serve as the foundation for a new unified approach to the whole of psychology, then it would seem to be necessary to expand the scope of at least some of Gibson's foundational concepts. Gibson's concepts, after all, were developed primarily to provide an account of perception. Gibson's theoretical approach and commitments are clear in the way that he structures his final book (Gibson, 1979). Gibson spends the first half of the book developing an account of what exists in the environment for organisms to perceive. (The use of the words 'organism' and 'animal' is deliberate. Gibson intends his approach to be general enough to apply to all of animal perception, not just human perception.) He begins with an account of the structure that exists in the world, and moves on to an account of how that structure gets conveyed via information in light to the visually perceiving organism. Gibson acknowledges that among 
the more functionally important parts of the environment are the other living organisms. For the most part, however, the focus is on a set of basic elements that enable perception: medium, substances, surfaces; events; energy arrays; persistence and change; optic flow fields; occluding edges; and so on (see the useful glossary of key terms in ecological optics in Appendix 1, Gibson, 1979, p. 307-309).

For those ecological psychologists interested in developing Gibson's approach and applying it to social phenomena, the problem is to identify how to extend Gibson's basic perception-oriented ontology in such a way that it can be usefully applied to behavior involving more than one acting organism. In other words, how can we turn Gibson's perception-oriented ontology into a social ontology, one that recognizes that the environment is populated with other actors and provides the necessary tools for characterizing the structures that arise in such a populated environment?

A number of proposals have been made about how to extend Gibson's ecological approach so that it can fruitfully be applied to the social (Heft 2020b). As a rule, these proposals tend to pick out one particular element of Gibson's perception-oriented ontology and to suggest that this particular theoretical element needs to be expanded or 'socialized'. Among the elements of Gibson's theory that researchers have suggested need to be modified so as to accommodate the social are: the concept of ecological information (for example, it is suggested that information can be shaped not only by laws of ecological physics but also by social convention, e.g. Barwise and Perry, 1983; Chemero, 2009; Bruineberg et al. 2019; Golonka, 2015; but see Baggs, 2015 for a dissenting view); the concept of affordances (Costall, 1995; Loveland, 1991; Pedersen and Bang, 2016; Rietveld and Kiverstein 2014); the concepts of skills and shared practices (Baggs et al., 2020; Baggs and Chemero, 2020b; van Dijk and Rietveld, 2017); and the boundaries of the organism itself (for example, it is sometimes suggested that the individual organism can become incorporated into a larger group unit or superorganism; Anderson et al. 2012; Marsh et al., 2009; Harrison and Richardson 2009; Riley et al., 2011). Finally, some theorists have proposed that we need to expand our account of the environment (Heft, 2001; Reed, 1996).

Plausibly, it may end up proving useful to adopt a combination of all of these proposals. What is unclear, at present, is how to fit all of these proposals together. What is needed is a framework to organize our thinking about social phenomena from an ecological starting point. This paper will be primarily concerned with the latter group of proposals, namely, those that focus on expanding the description of the kinds of structure that exist in the environment. The environment, after all, is where all social behavior happens. The ecological strategy, following Gibson, is to start with a description of the environment and to use this description as the basis of the psychology (Mace, 1977). We can hope that once we have a useful social description of the environment, we will have a framework in which the whole variety of social behavior will start to make sense.

The paper consists of a negative argument followed by a positive programmatic outline. The negative argument is specifically against the use of the term 'social affordance' as a means of expanding Gibson's perception-oriented ontology. This term has come to be widely used in the literature in recent years. I will argue that this term is unhelpful for the project of socializing Gibson's ecological approach. The term 'social affordance' offers only the illusion of explaining social phenomena in Gibsonian terms. It is based on a confusion of the perspective of the researcher with that of the animal. The term also creates an untenable dualism between the social and the nonsocial. I will suggest that the most sensible response to these problems is to adopt the position briefly entertained by Costall (1995), namely to adopt the claim that all affordances are social.

Next, in the positive, programmatic part of the paper, I present a schematic outline of the kinds of things that an extended ecological approach will need to be able to explain, once the description of the environment has been expanded to fully recognize the existence of other animals as actors, and to recognize the emergent multi-actor structures that arise in the environment as a result. To successfully extend the ecological approach in such a way that it can be usefully applied to behavior involving other individuals, it is necessary to describe environmental structures that arise at scales higher than that of the individual animal-environment system. It will be necessary to go beyond affordances and to develop tools (new ones and existing ones) for describing the environment at interpersonal or collective scales, incorporating symbolic and material culture.

\section{Is social affordance a useful category?}

If one is interested in developing a Gibsonian theory of the environment that fully incorporates the social, an obvious place to start is with the concept of affordances. The theory of affordances is a theory about how the meanings of things in the environment can be directly perceived by an animal (Gibson, 1979, 127). Among the things in an animal's environment, of course, are other animals. The theory of affordances implies that other animals are meaningful by virtue of what they afford to the perceiver (Gibson, 1979, 128).

On the face of it, it seems convenient to distinguish a sub-category of affordances to denote just those cases that involve other animals. The term 'social affordance' is sometimes used by researchers working within the ecological framework 
(McArthur and Baron, 1983; Valenti and Gold, 1991; Loveland, 1991; Marsh et al., 2006; Schmidt, 2007; Rietveld et al., 2019). The term is also coming to be commonly used by researchers working outside the ecological framework, within the broader cognitive sciences (e.g., Schilbach et al., 2013; Ramstead et al., 2016, Veissière et al., 2020; Brancazio, 2020). A succinct descriptive definition that captures the various ways in which the term 'social affordance' has been used is provided by de Carvalho (2020): 'Social affordances are possibilities for social interaction or possibilities for action that are shaped by social practices and norms.'

In my opinion, 'social affordance' is an unhelpful term. The increasing use of the term in recent years is an unfortunate trend. The term deserves to be subjected to some theoretical scrutiny and resistance. Fundamentally, the term is problematic because it confuses the perspective of the researcher with that of the animal. I will here highlight three ways in which this confusion of perspectives leads to unwelcome theoretical perplexities: (1) the term 'social affordance' implies the existence of a set of nonsocial affordances, but this creates a pernicious dualism which Gibson explicitly warned against; (2) the term encourages a commitment to an individualist or spectator view of social interaction, while ignoring interaction processes themselves; and (3) the term leads to a mischaracterization of higher-order or cultural processes. I will advise that in order to avoid these problems we should reject the term 'social affordances' altogether and adopt as a working hypothesis the claim that all affordances are social (i.e., all affordances are public).

\subsection{Objection 1: Material-social dualism}

The first objection is that referring to a class of 'social affordances' implies the existence of a complementary class of affordances that are non-social, or that are 'merely physical'. In other words, the term implies a material-social dualism. This is a dualism that Gibson sought to avoid. Gibson warned against creating a division between two environments, one material and one social (Gibson, 1979, 130):

It is a mistake to separate the natural environment from the artificial as if there were two environments; artifacts have to be manufactured from natural substances. It is also a mistake to separate the cultural environment from the natural environment, as if there were a world of mental products distinct from the world of material products.

The problem with dividing the environment into a 'material environment' and a 'cultural environment' is that although the distinction may seem to make sense from the perspective of the theorist who is thinking about these issues in the abstract, the division does not correspond to anything that is real for the organism. The organism does not experience two environments, but only one.

Organization theorists have recognized the problem of material-social dualism, and have made an effort to talk instead of the sociomaterial. In Wanda Orlikowsi's words, 'there is no social that is not also material, and no material that is not also social' (Orlikowski, 2007, 1437). Overcoming this material-social divide is likewise one of the concerns of the skilled intentionality framework, which draws on Orlikowski's work (van Dijk and Rietveld, 2017). The skilled intentionality approach is concerned in particular with human behavior (see Kiverstein, 2015, especially footnote 14: 'In my view all affordances that the human environment offers count as "social" because the affordances humans pick up on belong to an intersubjective shared reality and are bound up with normatively constrained social practices', my emphasis). Material-social dualism is, however, just as pernicious when it is applied to animal cognition as when it is applied to human cognition. There is every reason to try to understand the environment of non-human animals in sociomaterial terms as well.

\subsection{Objection 2: Ignoring interaction processes}

The second objection to the term 'social affordance' is that it implies a commitment to a classical understanding of action as something that the organism does to the environment: the action relation is theorized as a one-way causal flow from the organism out into its surroundings. This overlooks the fact that, for all actual living animals, there also exists a causal flow operating in the other direction. This is because the environment contains other animals that are liable to act back.

The fact that animate objects (i.e., other animals) in the environment can act back was again explicitly pointed out by Gibson $(1979,135)$ :

[Animate objects] are so different from ordinary objects that infants learn almost immediately to distinguish them from plants and nonliving things. When touched they touch back, when struck they strike back; in short, they interact with the observer and with one another. Behavior affords behavior, and the whole subject matter of psychology and of the social sciences can be thought of as an elaboration of this basic fact. 
The hallmark of interaction is that causation cannot be conceived in terms of a one-way flow outward from the individual. In interactive behavior, the individual's action is contingent on the ongoing action of the other. If the other stops acting in the appropriate way, the entire interaction breaks down. The reality and importance of interaction processes has long been recognized by developmental psychologists (Trevarthen and Aitken, 2001; Reddy, 2008). More recently, interaction has become a major focus of attention in embodied cognition research (Gallagher, 2020; Riley et al., 2011). This new focus on interaction processes is to be celebrated.

What should not be lost sight of, however, is that interactions always take place in a structured environment. It is not just the interaction that is structured; the environment in which the interaction takes place is structured too. The setting is sometimes lost sight of by interaction theorists. A recent paper by Brancazio (2020) attempts to combine affordance theory with the enactive theory of participatory sense-making, according to which interacting groups of organisms form autonomous self-sustaining units (De Jaegher and Di Paolo, 2007, Di Paolo et al., 2018). Brancazio avoids the term 'social affordance' on the grounds that it is too broadly defined. She introduces the term 'interpersonal affordance', which she defines as 'opportunities afforded by other agents.' Brancazio suggests that the perception of interpersonal affordances is 'different from the perception of environmental affordances' (Brancazio, 2020). The difference, according to Brancazio, is that perceiving interpersonal affordances involves the recognition of the agency of the other, which is absent for actions performed with reference to inanimate objects.

Whether or not affordance theory can be usefully combined with the theory of participatory sense-making, or enactive theory generally, remains an open question (Baggs and Chemero, 2021; Heft, 2020a). I note here only that the division between 'interpersonal affordances' and 'environmental affordances' would seem to be another expression of material-social dualism. Do interpersonal interactions not also take place in the environment? Brancazio, in her paper, makes several important points about identity and social power dynamics. These belong to human language-mediated behavior. To assume that interaction, in animals generally, is based on 'interpersonal affordances' is unhelpful, and only recreates the confusion of perspectives of researcher and subject that goes along with the rejected term 'social affordances'. The 'interpersonal affordances' term falls prey to the same problems and should also be rejected.

\subsection{Objection 3: Mischaracterizing higher-order processes}

The third objection is that trying to explain social and cultural systems by appealing to 'social affordances' is inappropriately reductive and encourages researchers to mischaracterize inherently interpersonal phenomena as intrapersonal ones. This is seen most clearly in recent proposals by predictive processing theorists. According to these theorists, symbol-involving behavior should be understood as being mediated by 'cultural affordances' (Ramstead et al., 2016; Veissière et al., 2020). One of the examples offered by these researchers is that of perceiving the meaning of a traffic signal. Ramstead et al. (2016) write (emphasis added): 'Red traffic lights, in virtue of convention (and law), 'mean' stop, and hence afford (and mandate) stopping - and this is made possible by the specifically human mastery of recursive inferences, both explicit and implicit, that agents make about other agents.' The first part of this sentence should be unobjectionable for Gibsonians, the second part less so.

The appeal to recursive inference-mentalizing or mindreading-implies that the meaning of the red traffic light is ultimately to be found inside the individual driver: the light derives its meaning from (its meaning 'is made possible by') the inferences made in the driver's disembodied mind (see Baggs and Chemero, 2020a).

But this is a curiously solipsistic view of the meaning of traffic signals. On a more ecological view, it would be better to say that the red traffic light gets it meaning from the system in which it is a component. A red traffic light means stop because it exists in an environment with a codified traffic system (this is acknowledged in the first part of the sentence quoted above from Ramstead et al., 2016): the traffic code, and its meaning, already exists in the populated environment even before you learn how to drive. It is true that traffic signs exist as 'merely legal obstacles [to locomotion] ... Their negative valence is based on potential legal consequences rather than potential collision' (Gibson and Crooks, 1938, 466). But potential legal consequences are a fact of the environment just as much as potential collisions are. (Compare this traffic light example with Gibson's description of a mailbox as something that 'affords letter-mailing to a letter-writing human in a community with a postal system'. Again, the postal system already exists before the individual actor comes along and perceives this particular mailbox. The meaning is not created by the individual's making of inferences. See Heft 2020b.)

For all of these terms - 'social affordances', 'cultural affordances', 'interpersonal affordances' — the relevant question is over what, if any, empirical purchase these terms can possibly be providing. In general, coming up with new terms is no substitute for the hard work of identifying behavioral phenomena that we wish to explain and identifying which aspects of the structure within the populated environment are relevant for explaining the behavior in question. 


\subsection{Why we should accept that all affordances are social}

In the abstract for his paper titled "Socializing affordances," Alan Costall poses this question (Costall, 1995, 467): 'what would ecological psychology stand to lose if all affordances were social?' In the paper itself, Costall distances himself from the strong version of the claim: 'My intention has not been to insist that all affordances are social, but to include the social squarely within the real' (478). In my opinion, the strong version of the claim deserves to be entertained carefully. I will argue that it deserves to be embraced. In fact, there is at least one plausible reading of the affordance concept according to which the claim that all affordances are social is true by definition.

To anticipate a potential confusion, it should be noted that the claim that 'all affordances are social' can be interpreted in more than one way. The claim could be interpreted, for instance, to mean that all actions necessarily involve multiple actors. This version of the claim is no doubt defensible in some sense (for instance, the Gaia theory of the earth's atmosphere as an organism-regulated system might imply that no behavior can truly be separated from the activities of other animals; Margulis and Lovelock, 1974). But I will not be defending this version of the claim here. The version of the claim that I wish to defend here might be paraphrased as 'all opportunities for action are public', or 'all opportunities for action are in principle observable'.

It is useful to set out the claim being defended alongside the possible alternatives. Logically, there are three possibilities: (1) no affordances are social; (2) some affordances are social; or (3) all affordances are social.

Let us also adopt a minimal criterion for identifying whether or not an affordance is 'social' (i.e., public):

Sociality criterion: An affordance, axiomatically, is an opportunity for action. We can say that an affordance is social if the performing of the action will directly impact, or will potentially impact, the perception-action activity of another organism or organisms, either now or in the future.

We can now compare the three possible options. Let us dispense immediately with the first possibility, namely that no affordances are social. This would only be true if animals never interacted with one another. The two live options are the second position, namely that some (but not all) affordances are social, and the third position, namely that all affordances are social.

One way to decide between these two is to ask: is there such a thing as a private affordance? Let us restrict ourselves initially to considering non-human animals. Of course, it is possible to imagine situations in which an animal acts alone, without immediately impacting the activity of any other animal. But is the animal's action thereby private? It is not private in the sense that it is impossible for an outside observer in principle to perceive the animal's activity. In fact, if there really are private affordances in animals, we will never discover them. The study of animal behavior relies on animals making public movements that can be observed by a scientist. At least for non-human animals, then, it seems quite plausible to say that all the opportunities for action that exist in the environment of a given animal are public (i.e. they are 'social' in the sense defined above).

The claim that all affordances are social may seem harder to defend once we consider human actors. Again, it is easy to think of situations in which performing an action has no immediate impact on other animals. This, however, does not invalidate the claim being defended here. For the purposes of the claim being defended here, it is only necessary to accept that all opportunities for action that are available to individual humans are in principle observable by a third party. The difficulty with humans is that we seem to spend a large amount of time 'in our heads'. We constantly talk to ourselves in an internal monologue in inner speech (Vygotsky, 1987), and we sometimes plan our movements mentally before actually performing them. In order to maintain that all affordances are social, it will be necessary to insist that thinking itself does not count as action, and to insist also that to think something is not to effect an affordance (although the thought may be about an affordance).

This may seem to impose an unnecessary limit on the scope of the affordance concept. I would disagree. The affordance concept cannot be expected to explain everything about mental life and experience. That is not what the concept is intended for. If everything in experience is an affordance, then the concept loses all explanatory power and becomes vacuous. The affordance concept is designed to perform a specific theoretical role. The concept is designed to explain how behavior can be directed meaningfully at things in the environment. (Researchers have recently proposed yet more sub-categories of affordance, such as 'epistemic affordances,' 'mental affordances,' 'mnemonic affordances'; see, e.g., Veissière et al., 2020. To the extent that such terms fail to refer to structure in the environment, these terms do not name affordances in the Gibsonian sense at all.)

Ultimately, then, the claim that all affordances are social follows trivially from a particular understanding of the affordance concept, and from a particular understanding of the environment. Importantly, it follows from an understanding of the environment as being populated with other animals. All of our actions are directed at a populated environment (Reed, 1996; Gibson, 1982, 411-412).. A positive program for dealing with social behavior within the Gibsonian 
POSTPRINT: ALL AFFORDANCES ARE SOCIAL

Action (when other animals are not co-present)

Rearranging the layout

Consuming resources

Creating resources

Leaving a trace

Recording something for a person to see later

Interaction (when other animals are co-present)

Other animals as objects

Self as object for others

What the other animal affords to oneself

What the world affords to the other animal

Using an object to perform an action on another animal

Coordinating with movements of other animals

What the world affords to us (as a collective)

Coordinating with event structure created by other animals

Directing another animal's attention to something

Pointing

Behavioral and symbolic culture

Actions learned within a particular cultural history

Naming

Predicating

Ascribing a 'correct use' to an object

Depicting

'Moves' within an existing cultural system (e.g. money)

Prohibited actions within a cultural system

Behavior settings

Scripts and routines

Role-taking

Rule-following

Identity

Material culture

Manufactured tools

Media of communication

Buildings and other built structures

Settlement layout

The climate

Table 1: Programmatic outline for studying behavior in a populated environment within the Gibsonian framework.

framework should start from a description of the kinds of structure that arise in an environment that is populated with multiple actors. We turn to this task now.

\section{The structure of the populated environment: a programmatic outline}

In this section, I present a programmatic outline of the kinds of environmental structure that must be accounted for under an ecological approach to perceiving and acting in a populated environment. The outline is programmatic in the sense that it presents a series of topics that we might hope will eventually be given a more thorough treatment within the ecological approach. The aim is to identify the ways in which it will be necessary to expand Gibson's initial perception-oriented description of the environment in order to accommodate the fact that the real environment is populated with other animals.

The outline is presented in the form of a list. It contains four headings, for four broad areas of study aimed at understanding behavior in a populated environment. The environment can be described in terms of: (1) action (actions by an individual directed at an environment in which other animals are not currently present); (2) interaction (actions directed at an environment in which other animals are currently present); (3) behavioral or symbolic culture; and (4) 
material culture. Note that these four labels do not denote four distinct environments, but four usefully distinguishable kinds of structure that exist in the one environment that exists.

The list is summarized in Table 1. The text in the rest of this section serves primarily to give examples of each of the items listed in the table. The reader may prefer to skip the examples and move on to the brief discussion at the end of the paper.

I have drawn on examples from the real world and from field studies, rather than from artificial laboratory situations. In the interests of avoiding anthropocentrism, I have drawn on non-human animal examples as far as possible. There are no doubt many types of multi-actor situations in the real world that do not fall neatly into any one of the categories listed below. For one thing, many real-world situations involve multiple animals engaged in multiple different, perhaps complementary, activities at the same time. The reader is invited to undertake the exercise of seeking real world examples that do not fit straightforwardly into the scheme presented here, or that might necessitate an expansion or revision of the scheme.

\subsection{Action (when other animals are not currently present)}

1. Rearranging the layout. Many actions, when carried out, result in a change in the layout of the inanimate parts of the environment. Example: an ant moves a particular leaf from location A to location B. Later a second ant comes along to location A, and the second ant does not find the original leaf there, for it has been moved. The layout has been re-arranged by the actions of the first ant.

2. Consuming resources. All animals necessarily consume resources from their environment. Actions that involve the consuming of resources change the environment in a special way. Such actions destroy some affordances in the environment. Example: a squirrel eats all the nuts from a birdfeeder. Those nuts now no longer exist anywhere in the environment, in ecological terms. In the extreme case, some actions destroy not only local token affordances, but an entire class of affordances. We humans have driven many species to extinction. Each extinct species represents a class of affordances that no longer exists in the world for any animal.

3. Creating resources. Animals not only destroy resources, but create resources too. The actions of earthworms change the chemical composition of the soil in such a way as to encourage plant growth above. This is likely beneficial to future generations of earthworms, and to other animals in the ecosystem (Nuutinen, 2011, Odling-Smee et al., 2003, 11). In mammals, predator species sometimes kill an animal and leave part of the carcass unconsumed. Scavenging species, such as vultures, who later come along to consume the carcass, are relying on resources that have been created by the predator. It's the circle of life.

4. Leaving a trace. Many mammal species leave identifying scent traces that serve a function of marking out territory (Ralls, 1971, Gosling, 1982). The animal that detects the scent has detected information about the local part of the environment, which might be glossed something like: "This place affords an aggressive encounter with another animal." Another kind of identifying trace is one that serves no function for the animal that leaves the trace, but that can nevertheless be picked up by another animal. Examples include footprints left in snow or scent trails picked up by hunting dogs.

5. Recording things for a specific person to see later. We humans leave traces of a special kind: we alter surfaces to display pictures and symbols. In childhood, we discover the 'fundamental graphic act' of making a lasting trace on a surface, for example by scribbling on a sheet of paper with a crayon (Gibson, 1966, 229). Initially we engage in this act for its own sake, simply observing the change in the surface as the crayon passes over it. Later we learn, or we are taught, that we can leave meaningful traces on a surface for another person to see later. The graphic act is thereby transformed into a communicative act. But here we are getting ahead of ourselves. Writing messages for another person involves language, which is part of the symbolic culture that exists in the environment. We will consider this below.

\subsection{Interaction (when other animals are currently present)}

1. Perceiving another animal or person (i.e. perceiving an animate object). The other animals in your environment are animate objects. They are 'detached objects with topologically closed surfaces, ... they change the shape of their surfaces while yet retaining the same fundamental shape' (Gibson, 1979, 135). In common with all other objects, the other animal affords a basic set of actions: observing, approaching, exploring, and so on.

2. Being an object for another animal or person. Reciprocally, you are an object in the environment of the other animal. You take up space. It is not possible for the other animal to occupy the space that you are currently occupying. And as an object, you afford to the other animal the same basic set of things that the other animal, being an object, affords to you—observing, approaching, exploring, etc. 
3. Seeing what the other animal affords. The other animal may have specific functional properties for the perceiver. These affordances may come in pairs-that is, there may exist a reciprocal affordance for the other animal (a mother affords something to the infant; the infant affords something to the mother). Alternatively, the affordance relation may go in only one direction (the jaw of a baleen whale may afford a nutrient-rich place for a barnacle to affix itself, while the whale itself may be indifferent to, and perhaps unaware of, the barnacle's presence).

4. Perceiving what the world affords for the other animal. We humans are sensitive to what the world affords to others. We restructure the environment for other humans, and even for non-humans (we provide dogs with chew toys, bees with artificial hives). It is likely that non-human animals, too, are sensitive to some information about what the environment affords to others. The cat that taps on the glass door and waits to be let into the house is perhaps sensitive to the fact that the door affords opening to the human (but not to the cat). In the wild, predators in pursuit must be sensitive to the flight behavior of their prey, and must thus be sensitive in some sense to what the environment affords to the fleeing prey.

5. Perceiving that an object or surface can be used to perform an action on another animal. Tool use in animals is well documented. Some animals make use of objects or surfaces in the environmental layout to perform actions on other animals in their environment. Standard examples are chimpanzees using sticks to fish for termites in a termite mound (Boesch and Boesch, 1990) and several species of bird that have been observed to drop shelled mollusks from a height onto a hard surface to crack open the shell (Zach, 1978).

6. Coordinating with the movements of another animal. There are many ways that animals can coordinate with the movements of other animals. The coordination can be reciprocal (as in play fighting); it can be rhythmic (as in the synchronized flashing of fireflies); it can be a local movement strategy that gives rise to an emergent group-level coordination pattern (as in geese flying in a ' $\mathrm{V}$ ' formation). In all of these cases, the dynamic structure that is created by the ongoing behavior itself creates the possibility for a given individual animal to coordinate in a particular way with the ongoing dynamics. This is presumably the kind of thing that Gibson was referring to when he suggested that '[b]ehavior affords behavior' (Gibson, 1979, 135).

7. Seeing what the world afford to us (collective action). Some actions cannot be performed by a single animal acting alone but require coordination between multiple animals. An example is group hunting behavior of the kind observed in several predator species. African wild dogs, for instance, are able, as a group, to hunt prey that it would be impossible for them to hunt individually. An individual dog weighs $20-25 \mathrm{~kg}$, but as a pack these animals hunt prey species weighing in the range 15-200 kg (Creel and Creel, 1995). This requires coordination and perhaps specialization (Estes and Goddard, 1967; Malcolm and Van Lawick, 1975).

8. Coordinating with event structure created by another animal. Any movement of the other animal's body creates event structure in the environment, and this structure can potentially be detected (you can see the other animal moving; you can detect the tremors of the ground as the elephant is walking). In addition, sometimes the other animal creates event structure that can be detected as such. Examples are birdsong, mating calls, or human music making (Searcy and Beecher, 2009; Yorzinski and Anoop, 2013)

9. Directing another animal's attention to something. Sometimes it is possible to direct the attention of another animal to some object or event in the environment that the other animal is not currently attending to. Many animal species exhibit vocal alarm calling behavior in response to predators (Owings and Morton. 1998). A well-known example is that vervet monkeys exhibit distinct alarm calls in response to distinct predators-leopards, eagles, and snakes (Seyfarth et al. 1980). An alarm call creates event structure in the environment of other monkeys that are close enough to hear the sound. An effect of this alarm calling behavior is that fellow vervet monkeys seek to flee to a safer location (Owings and Morton, 1997).

10. Pointing. Directing the attention of others deliberately is an important behavior in human infants. Children learn to point in order to direct the attention of others sometime around their first birthday (Leung and Rheingold, 1981). By learning to point, the child has discovered a technique for taking control of what they and the caregiver are jointly attending to. It has been suggested that this skilled control of joint attention is foundational to subsequent language learning (Tomasello et al., 2007).

\subsection{Behavioral and symbolic culture}

1. Actions learned within a particular cultural history. Among biologists the word 'culture' is applied generally to the behavior of many animal species, not just humans. The word refers to patterns of behavior that are transmitted between members of a group of animals (Jablonka and Lamb, 2005, 160). Standard examples of cultural transmission in animals are that blue tits in England learned from one another how to open foil-topped milk bottles, and that Japanese macaques learned from one another how to wash sand off potatoes (Aplin et al. 2013). Note that actualizing the affordance in these cases is simply a matter of manipulating an inanimate 
object in a particular way. But the manner of manipulation is learned from other animals, and hence is referred to as 'social learning.'

2. Naming. The human cultural environment is a linguistic environment through and through. To even begin to describe the human cultural environment, it is necessary to begin where language begins: in early childhood. Among the first real linguistic actions that children engage in, beyond merely making sounds, is the naming of things. In order for this process to get started there must be a caregiver present. Typically, the infant finds itself in an environment with a caregiver and with some other objects that are not the caregiver. We already noted above that, around its first birthday, the infant learns to point to things. The infant soon also learns that by uttering certain sound patterns-e.g., "dog"- - they can direct the attention of the caregiver to a particular object. Naming is an action that is afforded to the child, although it is not clear exactly what aspect of the environment it is that affords naming. Plausibly, the infant is responding to the whole situation, and the infant's action is directed at bringing about a particular three-way relation between itself, the caregiver, and the dog-the action is aimed at establishing joint attention (Baggs, 2015). This kind of one-word naming action in early infancy has been referred to as 'attentional action' (that is, an action designed to direct the attention of another person; Van den Herik, 2018), or 'indicational language' (Reed, 1996, 153).

3. Predicating. After the indicational stage, there follows a transition to the 'predicational' use of language, where the child not only indicates a topic (the dog), but comments on the topic too, using further linguistic structure to modify the meaning of the thing indicated (e.g., "the dog is sick"; see Reed, 1996, 161). This marks the start of a transition from language use as a strictly situated activity to language use as a symbolic activity. The child's use of language becomes 'ungrounded' from the immediate situation (Rączaszek-Leonardi et al. 2018). Symbolic use of language is qualitatively different from what is going on at the earlier indicational stage. The onset of symbolic thought allows the child to re-arrange their whole way of thinking: it gives the child a set of tools for directing their own attention-for steering their own stream of thought in an ongoing and structured way (Vygotsky, 1987; , Reed, 1996, 174-175).

4. Ascribing a 'correct use' to an object. Certain objects in the human environment have a 'preferred use' (Loveland, 1991; Costall 1995). We can distinguish here between two types of use pattern: habitual use, and symbolically-prescribed use. I may fall into the habit of using a fork to stir my tea. But the 'correct' object for this task is a teaspoon. A teaspoon has the 'canonical' affordance of stirring tea (Costall, 2012). Canonical affordances, in this second sense, are inherently language-involving. They belong to schematic perception, not literal (direct) perception (Gibson. 1950b, pp. 210-212).

5. Depicting. Humans have developed many techniques for presenting information to a viewer at second hand (Heft, 2017; Gibson, 1966, chapter 11).. Artificial perspective in paintings allows the artist to depict a scene to a potential viewer. Narrative language allows the speaker or the writer to depict a sequence of events to a listener or a reader. Techniques of depiction can involve the creation of lasting artifacts through the making of traces on a surface. The making of sound recordings performs an analogous role for the preserving of acoustic structure.

6. Actions possible only within an existing cultural system. Once a system of practices is in place, certain actions can become possible that would not be possible outside the system. The action is a 'move' within the system. A standard example is money. A banknote does not have any particular use value in itself-that is, as a mere piece of paper or synthetic polymer. It is, then, to some extent a mystery why we treat money as having any value at all (Epstein. 2015). The fact is that the banknote continues to function as money for only so long as the whole system of monetary exchange that supports it continues to operate.

7. Actions proh within an existing cultural system. Conversely, certain actions are proscribed by cultural convention. It is literally possible to walk around a grocery store eating the produce without paying for it, but culturally prescribed, and socially enforced norms require that we go through roundabout purchasing behaviors first (Gibson, 1950a).

8. Behavior settings. Human environments exhibit structure at multiple scales of organization: the individual, the kin group, the acquaintance network, the nation state (Hutchins, 2014). A useful scale for describing the day-to-day activity of humans is the scale of the behavior setting. A behavior setting is a higher order ecological unit that serves to structure the activity of the individuals present (Barker. 1968, Heft, 2001, 252). A behavior setting is not the same as a physical location. A table in one's house can serve to enable different behavior settings at different times. It can serve as a breakfast table at one time, a ping pong table at another time, a base for a papier-mâché volcano at a third time. The table, as an object, is the same in all three cases, and the same set of human participants may be involved at all three times, but the behavior is different in each case.

9. Scripts and routines. Within a behavior setting, behavior is typically patterned in characteristic ways. Participants sometimes follow a 'script' or 'routine'. A standard example is ordering food in a restaurant (Schank 
and Abelson, 1977). The server and the customer take turns uttering an often highly predictable series of phrases. (This is generally thought to require mental representations, but we need not accept that this is the case. All of the behavior here is public. It is observable by a third party - by a scientist, say, or by a child who happens to be at the table.) Routines are common in less formal settings, too. Children in western cultures are socialized into the routine of using utensils to eat food. During the socialization process the caregiver repeatedly structures the child's immediate environment, for example by realigning the spoon and the bow (Nonaka and Goldfield, 2018; Nonaka and Stoffregen, 2020).

10. Role-taking. Non-human animals sometimes exhibit polymorphic specialization. In ants there are 'workers', 'soldiers', and 'queens'. Each of these has a specific type of body. In human societies we adopt roles more flexibly and on a temporary basis. These roles can come with a symbolic label and with normative expectations. A person can be a 'caregiver,' a 'bus driver,' a 'wicket-keeper.' Enacting symbolic roles is an important part of pretend play in childhood (Fein, 1981).

11. Rule-following. To participate in some activities, it is necessary to learn a set of formal rules. In Piaget's system, learning to play games with rules comes after pretense play (Piaget, 1962; Fein, 1981). Rule-following is the basis of a good deal of adult behavior, such as in traffic systems.

12. Identity. A further distinctively symbolic way of seeing others is in terms of categories of identity: kin, tribe, ethnicity, gender, class, religion. Identity labels themselves are merely symbols, yet one's identity affects which affordances in the environment will be available and relevant, and which cultural practices one will learn to participate in. Identity is bound up with questions of power, status, duty, solidarity (Brancazio 2020).

\subsection{Material culture}

The list above is an attempt to identify some of the relevant structures that occur in an environment in which cultural processes are operating. It is useful to also enumerate the ways that lasting physical structures can arise through behavioral processes and can thereby create further possibilities for behavior. It is useful, that is, to recognize the importance of material culture in structuring the human environment (Baggs et al., 2019). Here we are describing the ways that the environment has been shaped by others, often in previous generations, in ways that are relevant to the animal now. Some of the things listed in this section are the kinds of things that archaeologists can potentially find long after the behavior that created the thing in question took place (Malafouris, 2013).

1. Manufactured tools. Tool manufacture (as opposed to merely using found objects as tools) is present to some extent in non-human animals, as in chimpanzees who prepare sticks by stripping leaves from a tree branch (Boesch and Boesch, 1990). The oldest objects created within the human lineage that are recognizable as manufactured tools and that have persisted to the present day are stones with a sharpened edge, thought to have been used for cutting. These tools were first created by Homo habilis some 2.5 million years ago (Donald, 1991. 110-112). This implies that manufactured tools have been present in our ancestral environment for a very long time. These sharpened stones predate the emergence of Homo sapiens by perhaps 2 million years. Other objects, such as string, clothing, wooden boats, or clay vessels persist for long enough to be used as tools in the day-to-day activity that occurs in a human environment (see Reed 1996, 120).

2. Media of communication. Written words, paintings, sculptures, photographs, sound recordings: these are all persisting structures that embody information and that thereby preserve that information in a format that is perceivable by future generations of humans. The storage of information in written form is particularly important for it marks the transition from the prehistoric to the historic.

3. Buildings and other built structures. Humans and other animals build structures in their environments. Spiders spin webs; birds construct nests. Beavers build lodges and dams, and in so doing they re-engineer the local water layout and establish a whole ecosystem (Naiman et al., 1986; Wright et al., 2002) A shelter of the sort built by humans-typically with a roof and walls-offers protection against sun and rain and predators. A building is also a place to store manufactured tools and other resources. A building is a setting in which other activities can take place: cooking, sleeping, nurturing, procreating. Other structures built by humans serve other special purposes: fences around a crop field keep animals from grazing the crop, a well offers a reliable local source of water, a bridge or a set of stepping stones offers a safe means of crossing a waterway.

4. Settlement layout. The built environment exhibits structure at multiple nested scales. When several buildings are built in proximity to one another, a higher order macro-structure emerges in the layout. The individual buildings can be built close together or they can be spaced apart; they can be built in parallel rows, in a spiral arrangement, etc. When the settlement is allowed to grow organically the emergent structure can be quite complex, as is the case in many old cities in Europe (Alexander, 1965, Hillier and Hanson, 1984). When the settlement layout is planned in advance the layout can be made to be simple, as when a city is built on 
a grid plan. Once the city is built, it can be very difficult to change the layout. For this reason, the street layout may retain much the same configuration for hundreds or even thousands of years, though the individual buildings may be torn down and replaced many times. Moreover, the layout of the city itself generates patterns of movement. A street segment that is well connected in the street network will naturally experience more traffic, because, being well connected, that segment of street is by definition relatively likely to lie on a path between any two arbitrary points in the settlement (Hillier et al., 1993). A segment of street that is strongly connected in the layout will receive a relatively high amount of foot traffic and is thus likely a good place to set up a trading stall; a segment of street that is weakly connected within the network will experience less traffic and may be a good place to carry out more private activities.

5. The earth's climate. It is odd to say that the climate of the earth is an artifact of material culture. Nevertheless, it is not unreasonable to say so. The carbon content of the atmosphere has increased dramatically as a result of human activity in recent decades. The most dramatic increases have been very recent indeed. Of all the fossil fuels that have been burned in human history, the majority has been burned in the last 30 years (Wallace-Wells, 2019).. Efforts to reverse anthropogenic global warming rely on our collectively treating the climate system precisely as an artifact that can be modified. This was pointed out by Gibson 40 years ago (Gibson, 1979, p. 130): 'There is only one world, however diverse, and all animals live in it, although we human animals have altered it to suit ourselves. We have done so wastefully, thoughtlessly, and, if we do not mend our ways, fatally.'

\section{Ecological ontology as social ontology}

The list above is an attempt to capture systematically at least some of the ways in which it is necessary to expand Gibson's initial perception-oriented description of the environment, once we have recognized that the environment is populated with other actors. Recall that, according to Reed, Gibson's ecological approach to perception was intended as the first step toward 'a new approach to the whole of psychology' (Reed, 1988, 2). In this paper I have been concerned specifically with the question of how Gibson's approach might eventually provide a foundation for a comprehensive ecological social psychology in particular (see also Heft, 2020b). I have been concerned, in other words, with the question of what should be included within a Gibsonian social ontology.

We can draw two conclusions from the discussion above. First, a distinction between social and nonsocial affordances is not tenable. The term 'social affordance' commits a category mistake. It divides the world up, from the perspective of the researcher and in a priori fashion, into 'social' and 'nonsocial' situations. It creates two environments. But affordances are not about the perspective of the researcher, they are about the perspective of the animal itself. From the perspective of the animal itself, there is only one environment. The 'social affordance' term should be rejected. A more promising strategy is to assume that everything that happens in the environment is public.

The second conclusion is that the affordance concept alone is not sufficient to explain social meanings and social phenomena. It is true that the populated environment offers, or affords, opportunities to the individual actor to act relative to both inanimate and animate objects. But it is also the case that the animate objects in the populated environment sometimes act back. The human environment, moreover, is filled not only with action-oriented meaning, but with symbolic meaning. An ecological social ontology will need to develop tools for dealing with this evolutionarily novel kind of meaning, a task that is as yet barely begun (Heft, 2017, Hutchins, 2014, Rączaszek-Leonardi et al., 2018).

The ecological approach to psychology is so called because it has always placed a primary focus on describing the structure of the environment. The ecological wager is that by starting with a description of the structure of the environment, our psychology will ultimately be built on a more secure foundation than would be the case had we started by describing the internal structure of the organism (Mace, 1977). This strategy has proved successful for re-thinking perception and action (Chemero, 2009; Turvey, 2018), and there is every reason to think that, if we avoid the pitfalls identified above, the approach can prove equally fruitful when applied to social psychology (Heft, 2020b).

There are two good reasons for adopting an ecological approach to social ontology, one methodological and one theoretical. The methodological reason is this. If we insist that the subject matter of social psychology is in fact public and observable, then it becomes possible to study social psychology as a natural science. If social meanings are public — if affordances are in principle observable by a third party - then a science of social cognition becomes empirically tractable. The ecological view of meaning allows us to avoid some perplexities that beset traditional theories of cognition. Under traditional accounts of meaning, such as Helmholtz's theory of meaning as inference, the status of social meaning is a conundrum. Traditional views of cognition are forced to confront the so-called problem of other minds. On the ecological understanding of meaning, according to which meaning is public (i.e. meaning is observable; it exists in the environment), these conundrums never arise in the first place. An ecological social psychology need not attempt to address such problems as the problem of other minds. The problem of other minds is revealed to be 
a pseudo-problem. Instead, an ecological social psychology can observe its subject matter directly. The working assumption is that the environmental structures that are implicated in the control of social behavior are in principle observable by the scientist. This is an attractive working assumption for methodological reasons because it greatly expands the scope of the ecological approach beyond more basic perception- and action-oriented behavior. Nor is this is not a wildly unorthodox assumption to make. The methodological assumption that meaning is public has a worthy history in psychology. It is an assumption that is adopted in the studies of animal behavior mentioned above. At one time the methodological assumption that the subject matter of psychology is public and observable was a widely accepted tenet in psychology more generally (Skinner, 1976, Heft, 2001, Charles, 2011; Barrett, 2019, Gibson, 1967a). We should revive it.

The second reason for adopting an ecological social ontology, meanwhile, is theoretical and is also ethical. Insisting that meaning is public provides a strategy for avoiding subjectivism in psychology. This was a central concern of Gibson's when he developed his ecological approach (Reed, 1988, chapter 3). Gibson found subjectivism in psychology to be morally unacceptable. He was convinced that a realist view of perception was necessary in order to provide a foundation for agreement between individuals. Gibson closes his 1966 book with these remarks (p. 321): 'The fact is that, although different men do not all use their senses in the same way, they can all use their senses in the same way. The basis for agreement among men exists in the available stimulus information. ... This book is dedicated to all persons who want to look for themselves.' (A similar remark appears in the final paragraph of Gibson's first book, in which he suggests that 'The ability to take the position of another person ... depends on being oriented in space'; Gibson, 1950b, p. 320.) For those of us who, like Gibson, would prefer to avoid subjectivism in our theorizing, a commitment to the view of meaning as a public commodity is perhaps the only viable starting point (Charles, 2011).

An ecological social ontology not only provides an empirically tractable way to pursue the study of the social. It also provides a way to avoid the view of the mind that has become commonsense in modern western philosophy, and that sees the individual as radically isolated from others and from the world itself. On the ecological view, social interaction and social cognition are possible because behavior is coordinated with respect to a single world that we are all living in. Meaning is public, and behavior is carried out within and relative to a meaningful environment that already has its own structure. Gibson's ecological ontology is already a social ontology. Gibson's strategy for investigating perception can quite naturally be applied to the investigation of social behavior (with apologies to Mace, 1977): ask not what's inside the animals' heads but what their heads are inside of.

\section{Acknowledgments}

I would like to thank the two reviewers for their insightful comments. I also thank Tony Chemero, Michael Anderson, and the members of the EMRG Lab social group at the University of Western Ontario for their comments on previous versions of the manuscript.

\section{References}

Alexander, C. (1965). A city is not a tree. Architectural Forum, 122(1):58-62.

Anderson, M. L., Richardson, M. J., and Chemero, A. (2012). Eroding the boundaries of cognition: Implications of embodiment. Topics in Cognitive Science, 4(4):717-730.

Aplin, L. M., Sheldon, B. C., and Morand-Ferron, J. (2013). Milk bottles revisited: social learning and individual variation in the blue tit, Cyanistes caeruleus. Animal Behaviour, 85(6):1225-1232.

Baggs, E. (2015). A radical empiricist theory of speaking: Linguistic meaning without conventions. Ecological Psychology, 27(3):251-264.

Baggs, E. and Chemero, A. (2020a). Thinking with other minds. Behavioral and Brain Sciences, 43(e90):24-25.

Baggs, E. and Chemero, A. (2020b). The third sense of environment. In Wagman, J. B. and Blau, J. J. C., editors, Perception as Information Detection: Reflections on Gibson's Ecological Approach to Visual Perception. Taylor \& Francis, New York, NY.

Baggs, E. and Chemero, A. (2021). Radical embodiment in two directions. Synthese, 198(Suppl 9):S2175-S2190.

Baggs, E., Raja, V., and Anderson, M. L. (2019). Culture in the world shapes culture in the head (and vice versa). Behavioral and Brain Sciences, 42(e172):16-17.

Baggs, E., Raja, V., and Anderson, M. L. (2020). Extended skill learning. Frontiers in Psychology, 11:1956.

Barker, R. G. (1968). Ecological Psychology. Stanford University Press, Stanford, California.

Barrett, L. (2019). Enactivism, pragmatism... behaviorism? Philosophical Studies, 176(3):807-818. 
POSTPRINT: AlL AFFORDANCES ARE SOCIAL

Barwise, J. and Perry, J. (1983). Situations and Attitudes. MIT Press, Cambridge, Massachusetts.

Boesch, C. and Boesch, H. (1990). Tool use and tool making in wild chimpanzees. Folia primatologica, 54(1-2):86-99.

Brancazio, N. (2020). Being perceived and being 'seen': Interpersonal affordances, agency, and selfhood. Frontiers in Psychology.

Bruineberg, J., Chemero, A., and Rietveld, E. (2019). General ecological information supports engagement with affordances for 'higher' cognition. Synthese, 196:5231-5251.

Charles, E. (2011). Ecological psychology and social psychology: It is Holt, or nothing! Integrative Psychological and Behavioral Science, 45(1):132-153.

Chemero, A. (2009). Radical Embodied Cognitive Science. MIT Press, Cambridge, Massachusetts.

Costall, A. (1995). Socializing affordances. Theory \& Psychology, 5(4):467-481.

Costall, A. (2012). Canonical affordances in context. Avant, 3(2):85-93.

Creel, S. and Creel, N. M. (1995). Communal hunting and pack size in African wild dogs, Lycaon pictus. Animal Behaviour, 50(5):1325-1339.

de Carvalho, E. M. (2020). Social affordance. In Vonk, J. and Shackelford, T., editors, Encyclopedia of Animal Cognition and Behavior, pages 1-4. Springer International Publishing, Cham.

De Jaegher, H. and Di Paolo, E. (2007). Participatory sense-making. Phenomenology and the Cognitive Sciences, 6(4):485-507.

Di Paolo, E. A., Cuffari, E. C., and De Jaegher, H. (2018). Linguistic Bodies: The Continuity Between Life and Language. MIT Press, Cambridge, Massachusetts.

Donald, M. (1991). Origins of the Modern Mind: Three stages in the evolution of culture and cognition. Harvard University Press, Cambridge, Massachusetts.

Epstein, B. (2015). The Ant Trap: Rebuilding the foundations of the social sciences. Oxford University Press,, Oxford.

Estes, R. D. and Goddard, J. (1967). Prey selection and hunting behavior of the African wild dog. The Journal of Wildlife Management, 31(1):52-70.

Fein, G. G. (1981). Pretend play in childhood: An integrative review. Child development, 52(4):1095-1118.

Gallagher, S. (2020). Action and Interaction. Oxford University Press, Oxford.

Gibson, J. J. (1950a). The implications of learning theory for social psychology. In Miller, J. G., editor, Experiments in Social Process, pages 149-167. McGraw-Hill, New York.

Gibson, J. J. (1950b). The Perception of the Visual World. Houghton Mifflin, Boston.

Gibson, J. J. (1966). The Senses Considered as Perceptual Systems. Houghton-Mifflin, Boston.

Gibson, J. J. (1967a). Autobiography. In Boring, E. G. and Lindsay, G., editors, A History of Psychology in Autobiography, volume 5, pages 125-143. Appleton-Century-Crofts, New York, NY.

Gibson, J. J. (1967b). New reasons for realism. Synthese, 17(1):162-172.

Gibson, J. J. (1979). The Ecological Approach to Visual Perception. Houghton-Mifflin, Boston.

Gibson, J. J. (1982). Notes on affordances. In Reed, E. and Jones, R., editors, Reasons for Realism: Selected essays of James J. Gibson, pages 401-418. Lawrence Erlbaum, Hillsdale, New Jersey.

Gibson, J. J. and Crooks, L. E. (1938). A theoretical field-analysis of automobile-driving. The American Journal of Psychology, 51(3):453-471.

Golonka, S. (2015). Laws and conventions in language-related behaviors. Ecological Psychology, 27(3):236-250.

Gosling, L. (1982). A reassessment of the function of scent marking in territories. Zeitschrift für Tierpsychologie, 60(2):89-118.

Harrison, S. J. and Richardson, M. J. (2009). Horsing around: Spontaneous four-legged coordination. Journal of Motor Behavior, 41(6):519-524.

Heft, H. (2001). Ecological Psychology in Context: James Gibson, Roger Barker, and the legacy of William James's radical empiricism. Lawrence Erlbaum Associates, Mahwah, NJ.

Heft, H. (2013). An ecological approach to psychology. Review of General Psychology, 17(2):162-167.

Heft, H. (2017). Perceptual information of "an entirely different order": The "cultural environment" in The Senses Considered as Perceptual Systems. Ecological Psychology, 29(2):122-145. 
Heft, H. (2020a). Ecological psychology and enaction theory: divergent groundings. Frontiers in Psychology, 11(991). Heft, H. (2020b). Ecological psychology as social psychology? Theory \& Psychology, 30(6):813-826.

Hillier, B. and Hanson, J. (1984). The Social Logic of Space. Cambridge university press, Cambridge.

Hillier, B., Penn, A., Hanson, J., Grajewski, T., and Xu, J. (1993). Natural movement: or, configuration and attraction in urban pedestrian movement. Environment and Planning B: planning and design, 20(1):29-66.

Hutchins, E. (2014). The cultural ecosystem of human cognition. Philosophical Psychology, 27(1):34-49.

Jablonka, E. and Lamb, M. J. (2005). Evolution in Four Dimensions: Genetic, epigenetic, behavioral, and symbolic variation in the history of life. MIT Press, Cambridge, Massachusetts.

Kiverstein, J. (2015). Empathy and the responsiveness to social affordances. Consciousness and Cognition, 36:532-542.

Leung, E. H. and Rheingold, H. L. (1981). Development of pointing as a social gesture. Developmental psychology, $17(2): 215$.

Loveland, K. A. (1991). Social affordances and interaction II: Autism and the affordances of the human environment. Ecological Psychology, 3(2):99-119.

Mace, W. (1977). James J. Gibson's strategy for perceiving: Ask not what's inside your head, but what your head's inside of. In Shaw, R. and Bransford, J., editors, Perceiving, Acting, and Knowing: Toward an Ecological Psychology, pages 43-65. Lawrence Erlbaum, Hillsdale, NJ.

Malafouris, L. (2013). How Things Shape the Mind. MIT Press, Cambridge, Massachusetts.

Malcolm, J. and Van Lawick, B. H. (1975). Notes on wild dogs (Lycaon pictus) hunting zebras. Mammalia, 39(2):231240.

Margulis, L. and Lovelock, J. E. (1974). Biological modulation of the Earth's atmosphere. Icarus, 21(4):471-489.

Marsh, K. L., Johnston, L., Richardson, M. J., and Schmidt, R. (2009). Toward a radically embodied, embedded social psychology. European Journal of Social Psychology, 39(7):1217-1225.

Marsh, K. L., Richardson, M. J., Baron, R. M., and Schmidt, R. (2006). Contrasting approaches to perceiving and acting with others. Ecological Psychology, 18(1):1-38.

McArthur, L. Z. and Baron, R. M. (1983). Toward an ecological theory of social perception. Psychological Review, 90(3):215-238.

Naiman, R. J., Melillo, J. M., and Hobbie, J. E. (1986). Ecosystem alteation of boreal forest streams by beaver (Castor canadensis). Ecology, 67(5):1254-1269.

Nonaka, T. and Goldfield, E. C. (2018). Mother-infant interaction in the emergence of a tool-using skill at mealtime: A process of affordance selection. Ecological Psychology, 30(3):278-298.

Nonaka, T. and Stoffregen, T. A. (2020). Social interaction in the emergence of toddler's mealtime spoon use. Developmental Psychobiology.

Nuutinen, V. (2011). The meek shall inherit the burrow: feedback in earthworm soil modification. In Karaca, A., editor, Biology of Earthworms, volume 24 of Soil Biology, pages 123-140. Springer.

Odling-Smee, F. J., Laland, K. N., and Feldman, M. W. (2003). Niche Construction: The Neglected Process in Evolution. Number 37 in Monographs in Population Biology. Princeton University Press, Princeton.

Orlikowski, W. J. (2007). Sociomaterial practices: Exploring technology at work. Organization studies, 28(9):14351448.

Owings, D. H. and Morton, E. S. (1997). The role of information in communication: An assessment/management approach. In Perspectives in Ethology, Vol. 12: Communication, pages 359-390. Springer, New York.

Owings, D. H. and Morton, E. S. (1998). Animal Vocal Communication: A new approach. Cambridge University Press, Cambridge.

Pedersen, S. and Bang, J. (2016). Historicizing affordance theory: A rendezvous between ecological psychology and cultural-historical activity theory. Theory \& Psychology, 26(6):731-750.

Piaget, J. (1962). Play, Dreams and Imitation in Childhood. WW Norton \& Co, New York.

Rączaszek-Leonardi, J., Nomikou, I., Rohlfing, K. J., and Deacon, T. W. (2018). Language development from an ecological perspective: Ecologically valid ways to abstract symbols. Ecological Psychology, 30(1):39-73.

Ralls, K. (1971). Mammalian scent marking. Science, 171(3970):443-449.

Ramstead, M. J., Veissière, S. P., and Kirmayer, L. J. (2016). Cultural affordances: Scaffolding local worlds through shared intentionality and regimes of attention. Frontiers in pPychology, 7:1090. 
POSTPRINT: AlL AFFORDANCES ARE SOCIAL

Reddy, V. (2008). How Infants Know Minds. Harvard University Press, Cambridge, Massachusetts.

Reed, E. S. (1988). James J. Gibson and the Psychology of Perception. Yale University Press, New Haven, Connecticut.

Reed, E. S. (1996). Encountering the World: Toward an ecological psychology. Oxford University Press, New York.

Rietveld, E. and Kiverstein, J. (2014). A rich landscape of affordances. Ecological Psychology, 26(4):325-352.

Rietveld, E., Rietveld, R., and Martens, J. (2019). Trusted strangers: social affordances for social cohesion. Phenomenology and the Cognitive Sciences, 18(1):299-316.

Riley, M. A., Richardson, M. J., Shockley, K., and Ramenzoni, V. C. (2011). Interpersonal synergies. Frontiers in Psychology, 2(38).

Schank, R. and Abelson, R. (1977). Script, Plans, Goals and Understanding: An Inquiry into Human Knowledge Structures. Lawrence Erlbaum Associates, Hillsdale, New Jersey.

Schilbach, L., Timmermans, B., Reddy, V., Costall, A., Bente, G., Schlicht, T., and Vogeley, K. (2013). Toward a second-person neuroscience. Behavioral and Brain Sciences, 36(4):393-462.

Schmidt, R. C. (2007). Scaffolds for social meaning. Ecological psychology, 19(2):137-151.

Searcy, W. A. and Beecher, M. D. (2009). Song as an aggressive signal in songbirds. Animal Behaviour, 78(6):12811292.

Seyfarth, R. M., Cheney, D. L., and Marler, P. (1980). Monkey responses to three different alarm calls: Evidence of predator classification and semantic communication. Science, 210(4471):801-803.

Skinner, B. F. (1976). About Behaviorism. Random House, New York, NY.

Tomasello, M., Carpenter, M., and Liszkowski, U. (2007). A new look at infant pointing. Child development, 78(3):705-722.

Trevarthen, C. and Aitken, K. J. (2001). Infant intersubjectivity: Research, theory, and clinical applications. Journal of Child Psychology and Psychiatry, 42(1):3-48.

Turvey, M. T. (2018). Lectures on Perception: An Ecological Perspective. Routledge, New York.

Valenti, S. S. and Gold, J. M. (1991). Social affordances and interaction I: Introduction. Ecological Psychology, 3(2):77-98.

Van den Herik, J. C. (2018). Attentional actions - an ecological-enactive account of utterances of concrete words. Psychology of Language and Communication, 22(1):90-123.

van Dijk, L. and Rietveld, E. (2017). Foregrounding sociomaterial practice in our understanding of affordances: The skilled intentionality framework. Frontiers in psychology, 7:1969.

Veissière, S. P., Constant, A., Ramstead, M. J., Friston, K. J., and Kirmayer, L. J. (2020). Thinking through other minds: A variational approach to cognition and culture. Behavioral and Brain Sciences, 43(e90):1-75.

Vygotsky, L. S. (1987). Thinking and Speech. In Rieber, R. W. and Carton, A. S., editors, The Collected Works of L. S. Vygotsky, volume 1. Plenum Press, New York.

Wallace-Wells, D. (2019). The Uninhabitable Earth: Life after Warming. Penguin, New York.

Wright, J. P., Jones, C. G., and Flecker, A. S. (2002). An ecosystem engineer, the beaver, increases species richness at the landscape scale. Oecologia, 132(1):96-101.

Yorzinski, J. L. and Anoop, K. (2013). Peacock copulation calls attract distant females. Behaviour, 150(1):61-74.

Zach, R. (1978). Selection and dropping of whelks by northwestern crows. Behaviour, 67(1-2):134-147. 